\title{
Schweiz wird Mitglied bei der IHTSDO
}

\section{Johannes Gnägi}

Wissenschaftlicher Mitarbeiter "eHealth Suisse»

\section{Ausgangslage}

In Zukunft werden Dokumente, die für alle Behandelnden eines Patienten wichtig sein können, im elektronischen Patientendossier abgelegt. Diese Informationen können unstrukturiert sein (zum Beispiel als PDF-Dokument) oder strukturiert (zum Beispiel in einer XMLStruktur). "Unstrukturiert» bedeutet, dass in einem Austrittsbericht die Informationen zu Diagnosen, Laborwerten oder Medikamenten nur von Menschen gelesen werden. Sind die Informationen dagegen "strukturiert» verfügbar, dann können die Inhalte vom Computer erkannt und automatisch weiterverarbeitet werden. Im oben erwähnten Beispiel mit dem Austrittsbericht können die Informationen aus dem IT-System des Spitals direkt ins IT-System einer Arztpraxis übernommen werden - ohne fehleranfällige Übertragung von Hand.

Damit dies möglich wird, muss neben der technischen auch die semantische Interoperabilität sichergestellt werden. Das heisst: Zwischen dem Sender und dem Empfänger muss geklärt werden, wie eine Information bezeichnet wird (zum Beispiel mit einem Code). Für Diagnosen hat sich dafür weltweit die ICD-Klassifikation der WHO durchgesetzt. In anderen Domänen haben sich andere nationale oder internationale semantische Standards etabliert - in der Schweiz zum Beispiel CHOP für die Klassifikation von Operationen.

Auch im Pflegebereich gibt es viele Aktivitäten rund um Terminologien wie ICNP (International Classification for Nursing Practice) oder NANDA (North American Nursing Diagnosis Association).

In der Schweiz erachtet es die «Expertengruppe Semantik» von «eHealth Suisse» als zwingende Option, die diversen Standards mit SNOMED CT als Referenzterminologie zusammenzubringen. Generell darf festgehalten werden, dass die mit dem Thema Semantik vertrauten Fachleute aus dem Umfeld der Ärzteschaft, der Apotheker und der Pflege den Einstieg in die SNOMEDCT-Welt begrüssen.

Ein umfassender semantischer Standard, der alle medizinischen Fachgebiete abdeckt, existiert bis heute nicht. Dem Anspruch am nächsten kommt SNOMED
CT (Systematized Nomenclature of Human and Veterinary Medicine-Clinical Terms).

Vor diesem Hintergrund und basierend auf der Vereinbarung mit IHTSDO (International Health Terminology Standards Development Organisation) enthalten inzwischen drei von "eHealth Suisse" verabschiedete nationale Empfehlungen diverse Codes von SNOMED CT:

- Austauschformat "Elektronisches Impfdossier» (Dokument des zukünftigen ePatientendossiers);

- Austauschformat «Meldepflichtige Laborbefunde der Schweiz» für die elektronische Meldung von Erregernachweisen an die Sektion Meldesysteme des BAG;

- Austauschformat «Laborbefunde im Transplantationsprozess» für die elektronische Übermittlung von Laborbefunden an das Swiss Organ Allocation System (SOAS).

\section{Nutzung SNOMED CT in epSOS/EXPAND}

Einen weiteren Anwendungsfall verfolgen die EU-Mitgliedstaaten im grenzüberschreitenden Datenaustausch. Im europäischen epSOS-Projekt (European Patients - Smart open Services), an dem die Schweiz mit dem Kanton Genf teilgenommen hat, wurde dieser Ansatz erfolgreich getestet. Das in epSOS definierte «Patient Summary» konnte in der jeweiligen Landessprache genutzt werden, weil die medizinischen Informationen mit SNOMED CT eindeutig definiert waren. Für die Inhalte war in jedem Land eine standardisierte Übersetzung in der Zielsprache verfügbar. Somit konnte zum Beispiel ein im Unispital Genf erstelltes französisches "Patient Summary» in Lissabon in portugiesischer Sprache gelesen werden. Aufgrund der guten Erfahrungen hat die Europäische Kommission mit IHTSDO eine Vereinbarung abgeschlossen, wonach die in epSOS festgelegten Codes in den EU-Mitgliedstaaten auch ohne nationale Lizenz verwendet werden können.

Dieser Standard kann als eigene Sprache verstanden werden, mit dem medizinische Sachverhalte sehr genau und differenziert beschrieben werden können. 
Bei der Definition von ersten Inhalten für das elektronische Patientendossier (sogenannte "Austauschformate») sind die Grenzen der heute verfügbaren Terminologien sichtbar geworden. So konnten zum Beispiel die im Schweizerischen Impfplan geforderten Informationen zu Allergien im Austauschformat zum elektronischen Impfdossier nur mit Codes von SNOMED CT korrekt abgebildet werden. Vor diesem Hintergrund haben das BAG und das Koordinationsorgan «eHealth Suisse» im September 2013 eine Vereinbarung unterschrieben für den schrittweisen Einstieg der Schweiz in die Nutzung von SNOMED CT. Vertragspartner ist die IHTSDO mit Sitz in London (UK). Diese pflegt die Terminologie SNOMED CT und entwickelt sie weiter. International gibt es einen Trend, SNOMED CT als Referenzterminologie zwischen bestehenden semantischen Standards zu nutzen. Dies mit der Idee, dass die Akteure weiterhin mit ihren Fachstandards arbeiten können, diese aber untereinander via SNOMED CT semantisch interoperabel sind. Deshalb hat IHTSDO Partnerschaften etabliert: mit der WHO im Kontext der Weiterentwicklung der ICD-Klassifikation zu ICD-11 oder mit dem Regenstrief Institute zu international einheitlichen Laborinformationen mit LOINC-Codes.

\section{Mitgliedschaft IHTSDO}

Seit 2016 verfügt die Schweiz über eine Volllizenz von SNOMED CT über die Mitgliedschaft bei IHTSDO. Die IHTSDO ist eine internationale Non-Profit-Organisation. Sie hält die Rechte für SNOMED CT. Sie ist verantwortlich für die dauernde Pflege, Weiterentwicklung, Qualitätssicherung und Herausgabe von SNOMED CT und die Harmonisierung mit anderen terminologischen Standards (zum Beispiel bei ICD-11). Mit dem Beitritt der Schweiz kann SNOMED CT von allen Akteuren (Anwender und Hersteller) schweizweit kostenlos eingesetzt werden.

\section{National Release Center}

Als Ansprechpartner in der Schweiz dient das National Release Center (NRC), welches von "eHealth Suisse» verwaltet wird. Das NRC ist der Kontaktpunkt für die Kommunikation mit der IHTSDO und anderen Mitgliedern. Für die Schweiz verwaltet es die Lizenzierung und Verwendung von SNOMED CT und dient als zentrale Anlaufstelle für die verschiedenen Stakeholder wie Anwender und Hersteller von IT-Systemen, die SNOMED CT einsetzen wollen. Die grundlegenden Aufgaben sind:

- Verwaltung und Herausgabe von Lizenzen für Nutzer (Firmen etc.);

- Verteilen der internationalen Version von SNOMED CT sowie Updates;

- Entgegennahme von Change-Requests für Inhalte von SNOMED CT.

\section{Registrierung}

Damit SNOMED CT verwendet werden kann, ist eine Registrierung beim NRC notwendig. Um sich für die Verwendung von SNOMED CT zu registrieren, sind folgende Schritte nötig:

1. Registration auf dem «Member Licensing \& Distribution Service» (MLDS);

2. Erstellen und Aktivieren eines Accounts, Bereitstellung der nötigen Informationen;

3. Akzeptieren der SNOMED-CT-Lizenzvereinbarungen (Affiliate License Agreement);

4. Download von SNOMED CT über den Downloadlink, welcher ca. 4-5 Arbeitstage nach Aktivierung zugeschickt wird.

Für Fragen und Anliegen steht Ihnen auch das National Release Center Schweiz zur Verfügung. 